\title{
Announcing the Winners of the 2010 International E-Learning Awards!
}

We are pleased to announce the winners of the 2010 International E-Learning Awards! Awards were given in both the academic and business/professional categories for e-learning, blended learning, and mobile learning. Submissions were received from 26 countries overall.

Congratulations to all our winners, and look for the Call for Submissions for the 2011 International ELearning Awards coming soon, via e-mail and on the International E-Learning Association web site at http://www.ielassoc.org !

\section{International E-Learning Awards - Award Recipients}

\section{Winner, Academic E-Learning}

"The IR Model: Dissertation Module"

Beyond Distance Research Alliance, University of Leicester, Leicester, Leicestershire, UK

Representative: Simon Kear

\section{Winner, Academic Blended Learning}

"Space Trainees"

MediaCity, Vasa, Finland

Representative: Simon Staffans

\section{Winner, Business/Professional E-Learning \\ "ePhysicianHealth.com"}

Faculty Wellness Program, Faculty of Medicine, University of Ottawa, Ottawa, Ontario, Canada

Representatives: Derek Puddester and Emma Stodel

Winner, Business/Professional Mobile Learning

“Operation Numerika” on Nintendo DS

Epic Performance Improvement Ltd, Brighton, East Sussex, United Kingdom

Representative: Alec Keith

\section{Winner, Business/Professional Blended Learning}

"Brand Edge -marketing capability program delivering brand growth in GSK worldwide", GlaxoSmithKline with agency partners Oxford Strategic Marketing (consulting \& capability building), Push Thinking (internal communications), and TRiBECA (eLearning, knowledge architecture, web development)

Representative: André Moa, TRiBECA, London, UK

\section{Runner-Up, Academic E-Learning}

"Distributed Software Development Course"

University of Zagreb, Faculty of Electrical Engineering and Computing, Zagreb, Croatia

Representative: Mario Zagar

\section{Runner-Up, Academic E-Learning}

"MySelf e-Portfolio"

University of Piraeus, Department of Digital Systems, Athens, Greece

Representatives: Fotini Paraskeva and Aikaterini Alexiou

\section{Runner-Up, Academic Blended Learning}

"Managing and Organizing Information" Course Website Institute of Public Administration, Riyadh, Saudi Arabia Representative: Dr. Ajlan M. Alshehri

\section{Runner-Up, Academic Blended Learning}


“Web 2.0 Knowledge Map”, ZLW - Center for Learning and Knowledge Management \& IMA - Institute of Information Management in Mechanical Engineering \& ifU - Institute for Management Cybernetics, RWTH Aachen University, Aachen, Germany

Representative: Sabina Jeschke

\section{Runner-Up, Business/Professional E-Learning}

"CoachTrue Elite"

World Anti-Doping Agency and Web Courseworks, Montreal, Canada and Madison, WI, USA

Representative: Aileen Zhang

\section{Runner-Up, Business/Professional E-Learning}

"VISTA Civil Rights and Responsibilities Course"

Education Northwest, Portland, Oregon, USA

Representative: Angela Nicholas

\section{Honorable Mention, Academic E-Learning}

"Fish - Market”, Pädagogische Hochschule Salzburg, Salzburg, Austria

Representative: Uwe Gutwirth

\section{Honorable Mention, Academic E-Learning}

"MUS241: Music Appreciation"

The University of North Carolina at Greensboro Division of Continual Learning,

Greensboro, North Carolina, USA

Representative: Coventry Kessler

\section{Congratulations to all of our 2010 winners, the quality of submissions was excellent!}

David Guralnick

President

International E-Learning Association 